
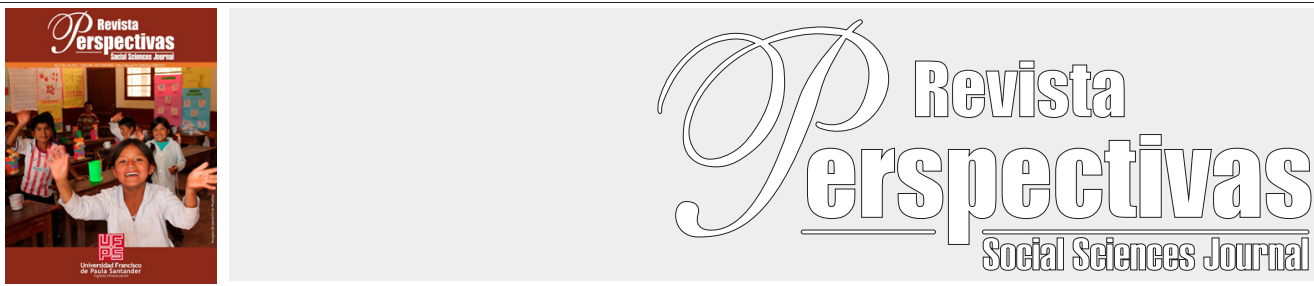

Artículo Original

https://doi.org/10.22463/25909215.3011

\title{
Libertad de prensa y diversidad: no existe libertad de prensa sin diversidad e igualdad de géneros
}

\section{Press freedom and diversity: no press freedom without diversity and gender equality}

Lorena Vanesa Elizalde ${ }^{1}$

${ }^{1}$ Mrofesora de Posgrado en la Universidad Internacional de Valencia, España. Investigadora Universidad Nacional de La Plata- Cátedra de Cultura Jurídica Argentina. +5492214112364.draelizalde@gmail.com

Cómo citar: L.V. Elizalde, "Libertad de prensa y diversidad: no existe libertad de prensa sin diversidad e igualdad de géneros". Perspectivas, vol. 6, no. 2, pp. 99-108, 2021.

Recibido: February 02, 2021; Aprobado: May 15, 2021.

\begin{tabular}{ll}
\hline & RESUMEN \\
\hline Palabras clave: & En los tiempos sin precedentes que el mundo se encuentra viviendo, debido a la actual pandemia del \\
Covid-19. Libertad de & COVID-19, se ha venido visibilizado una mayor presencia de tareas fuera del hogar que antes realizaban \\
Expresión. Cultura. & solo los hombres. Dígase que son las mujeres, mujeres activistas, feministas, comunidad LGBTI+ entre \\
Opinión. Periodismo. & ser cumplen un rol protagónico. Esto evidencia las enormes desigualdades que persisten a la hora de \\
Mujer. Géneros. & y debate públicos. Si bien, América han venido superando ciertas barreras, con alguna integración de las \\
& minorías y de ciertos grupos culturales, como así también de inclusión de diversidad de género a nivel \\
& legislativo y jurisprudencial. En la práctica hay muchos países, que manipulados por grupo de poderes y \\
& sectores empresariales poderosos se resisten a brindar los espacios y legitimar las opiniones de los géneros. \\
& En una de estas ramas, y se considera la más importante por su difusión e impacto, es la prensa, los medios \\
& de comunicación masivos, redes sociales, entre otros. Esto no es ni más ni menos que el reflejo, del recorte \\
& esas condiciones de igualdad, de no discriminación, de representatividad, que las diferentes legislaciones \\
intentan proteger en su pleno ejercicio.
\end{tabular}

\begin{tabular}{ll}
\hline ABSTRACT \\
\hline Keywords: & $\begin{array}{l}\text { In the unprecedented times that the world is living, due to the current COVID-19 pandemic, a greater presence } \\
\text { of tasks outside the home than previously performed only by men has become visible. Tell yourself that women, } \\
\text { women activists, feminists, the LGBTIQ + community, among others, play a leading role. This shows the enormous }\end{array}$ \\
$\begin{array}{l}\text { Covid-19. Freedom of } \\
\text { inequalities that persist when gender is represented in spaces for citizen participation, centers that generate opinion } \\
\text { and public debate. Although, America has been overcoming certain barriers, with some integration of minorities } \\
\text { and certain cultural groups, as well as the inclusion of gender diversity at the legislative and jurisprudential level. } \\
\text { Gender. }\end{array}$ & $\begin{array}{l}\text { In practice, there are many countries that, manipulated by groups of powers and powerful business sectors, are } \\
\text { reluctant to provide the spaces and legitimize the opinions of the genders. In one of these branches, and it is } \\
\text { considered the most important for its dissemination and impact, it is the press, the mass media, social networks, } \\
\text { among others. This is neither more nor less than the reflection, of the reduction of those conditions of equality, of } \\
\text { non-discrimination, of representativeness, that the different legislations try to protect in their full exercise. }\end{array}$
\end{tabular}




\section{Introducción}

El recorte de la libertad de expresión, se observa en los debates u opiniones que construyen en los medios de prensa. Esto es, por ejemplo, en temas tales como la legalización de la prostitución, aborto seguro, igualdad de género, matrimonio igualitario, adopción igualitaria, fertilización asistida, cambio de género, edición genética, entre otros.

Se visualizan en estos espacios, sean, televisivos, digitales, virtuales, un recorte de la libertad de opinión, libertad de prensa y en sí de libertad de expresión. Estos espacios son copados por la opresión y la invisibilización de las verdaderas actoras y actores de estos debates, esto es las mujeres, las mujeres activistas, las feministas, los sindicatos de meretrices, la comunidad LGBTIQ+ por mencionar algunos.

En estos espacios no existe libertad de opinión irrestricta, por ende, no hay libertad de prensa. No sólo porque no hay un reconocimiento del rol de la mujer periodista, sino que siempre se tiende, por los grandes medios, a callar e invisibilizar las voces de los diferentes, a silenciar a los que piensan y ven distinta la vida, se tiende a opacar las versiones de quienes realmente viven dramáticamente las situaciones que están o se encuentran en el debate público.

La visibilización de las estrategias de los medios opresores, de la censura de ideas, de la supresión de contenidos, eliminando cuerpos, denostando identidades, destruyendo el verdadero discurso de los protagonistas en el espacio radial, televisivo y digital se encuentra presente en todos los contextos y en todos los países. Si bien, con diferentes trazos y pinceladas, se detectan siempre, sean en América, en Europa, en Asia, en Países islámicos, entre otros.

Los contextos machistas, hetero-patriarcales, paternalistas, conservadores y de derecha son los que enarbolan las banderas de los debates públicos y construyen la arena pública y la agenda política, a los efectos de generar opinión, generar ideas, emociones, decisiones y posiciones en la sociedad. Esto denota el fin con el que los mismos lo hacen, esto es ir en contra la igualdad de los géneros, rechazo de la lucha feminista entre otras motivaciones que iremos sombreando.

La mirada de la sociedad, para con estos medios, poderes, o prensa, solo se puede de-construir y construir con la educación, educación plural y laica de acceso igualitario a toda la comunidad. El deconstruir lo construido por cientos de años debe llevarnos a en algún punto comenzar a romper con las ideas machistas de que todo lo que no es categoría "hombre" debe estar en el ambiente privado, encerrado en el hogar, que no es válido en la esfera pública las opiniones de lo que no entra en esa categoría binaria.

Las opiniones de las mujeres, las mujeres activistas, las feministas, las organizaciones pro mujer, la comunidad LGBTIQ+, comunidad afroamericana, latina, entre otras, son tan válidas en la prensa y en los medios como cualquier otra que brinde un hombre sexo/género binario.

Todas aquellas opiniones que no son brindadas por un agente que no se identifique con un hombre, en la mirada desde la interseccionalidad, se les exige mayor dato, fundamento, argumento académico. Las opiniones diferentes, miradas y percepciones distintas, caen en un saco roto, y nunca pasan a ocupar el espacio público con legitimidad.

\section{Mujeres y Periodismo.}

Intentaremos explorar un poco estas tres dimensiones de las mujeres y el periodismo.

pública?

¿Cuáles son los reproches típicos que se les hace a estas voces cuando intentan ocupar el espacio público de opinión, en la prensa? 
¿A que se enfrentan las mujeres periodistas cuando deciden alzar la voz públicamente?

\section{Datos y hechos.}

Para brindar algunos datos el cuarenta y nueve y cinco por ciento población mundial en el mundo son mujeres. El cincuenta por ciento carrera de periodismo nivel mundial, son mujeres. El veintitrés por ciento trabajan como Jefes de redacción, son mujeres y son minoría.

Es del todo claro, para nosotras las mujeres, que la vivencia femenina puede abrir abanicos importantes de temas que los hombres no tienen ni siquiera en mente simplemente porque son otros, ni mejores ni peores, otros con otra forma de pensar y con lentes de otros colores.

Que en las redacciones de medios de comunicación sean mayoría hombres no es una cuestión mínima. Los jefes de redacción son quienes forman, integran y defienden un tipo de agenda social de un país, dejando afuera temas que no se hablan y que se convierten en temas desechados.

Las mujeres no estén en los centros de opinión y de poder de los medios de comunicación tuerta, analiza la sociedad y la realidad con una mirada defectuosa.

No mencionamos nada nuevo, si entendemos que el reparto en los medios de prensa, es desigual e injusto. Que más allá de las marchas feministas, que intentan que romper los estereotipos, esta explosión de visibilización femenina, va a llevar muchos años a la sombra, sino se debate hoy, el cómo y cuándo debe ocurrir el futuro rompimiento del famoso techo de cristal.

Solo el veinticuatro por ciento de las personas entrevistadas, escuchadas, vistas, o sobre las que se ha escrito en los medios de comunicación escritos y audiovisuales son mujeres, mientras que el setenta y seis por ciento son hombres.

Solo el trece por ciento de las noticias ponen a las mujeres en primer plano. Menos de uno de cada cinco especialistas entrevistados por los medios de comunicación son mujeres.

Las mujeres periodistas hacen el treinta y siete por ciento de las noticias que se difunden. En sus reportajes, estas desafían los estereotipos de género dos veces más que sus colegas de sexo masculino.

El sesenta y cinco por ciento, de los puestos de trabajo en las áreas de producción y diseño pertenecen a los hombres, y solo un treinta y cuatro por ciento a las mujeres.

En el cuarenta y cuatro de los países donde se realizó este estudio se constató una representación insuficiente de la mujer en los medios de comunicación.

Pasemos a los desafíos que se encuentran las mujeres a la hora de ingresar a ejercer su rol o de desempeñarlo.

Sin dejar de mencionar lo obvio, hoy por hoy internet, la red, lo online, como les guste llamarlo, es el lugar que abre más puertas a la libertad de expresión.

Casualmente en el informe de periodismo frente al sexismo de "Reporteros sin Fronteras" se señala a Internet como el lugar más peligroso para las mujeres periodistas. El solo hecho de ser mujer implica ser blanco de críticas, acoso y ataques. Las cuales dan en consecuencia el cierre de sus cuentas de internet con el resultado de silenciar a estas voces. Las mujeres periodistas están expuestas a mayor violencia sexista y sexual que sus compañeros hombres.

Primeramente, vamos a ver un poco de hechos y cifras que han sido obtenidas conforme al informe 
de la Unesco respecto del trato hacia las mujeres periodistas, las barreras culturales y las luchas que tienen que padecer las mujeres para poder ejercer su profesión en los medios.

El setenta y tres por ciento de las mujeres periodistas ha sufrido alguna vez acoso en línea relacionado con su trabajo. Una nueva investigación publicada por la Unesco reveló que las periodistas reciben a menudo mensajes "misóginos coordinados" y que van desde el "lenguaje de odio hasta amenazas de violencia sexual o física."

En los últimos años la Relatoría Especial de la $\mathrm{CIDH}$, junto con la comunidad internacional, ha realizado esfuerzos para llamar la atención respecto de las formas de violencia contra las mujeres basada en su género a las que se enfrentan las periodistas en el ejercicio de su profesión, así como al impacto desproporcionado que tienen ciertas formas de discriminación en su trabajo. En especial, la Relatoría Especial ha llamado la atención respecto al incremento de actos de violencia contra mujeres periodistas, incluidos los asesinatos, la violencia sexual y la violencia en línea. Además de ser objeto de la amplia gama de violaciones de los derechos humanos que afectan a los periodistas en general, "las mujeres periodistas de manera desproporcionada y habitual se enfrentan a la violencia por razón de género en el lugar de trabajo y sobre el terreno."

De acuerdo con Atziri Ávila, existe cero tolerancia y aceptación frente a quienes deciden romper el silencio. La respuesta inmediata, mediocre y rapaz de los agresores de comunicadoras y periodistas son ataques de todo tipo: montajes fotográficos, campañas de difamación de su trabajo y persona, calumnias que aluden a su vida personal, amenazas de violación sexual, amenazas de muerte, amenazas contra sus hijos e hijas, agresiones físicas, robo de material, desvalorización de su labor, allanamientos a sus hogares, invasión a sus espacios más íntimos o asesinatos, como fue el caso de la periodista Miroslava Breach, asesinada el veintitres de marzo 2017. Miroslava dio voz a los pueblos Wirrarika de Chihuahua ante la invasión de empresas en su territorio, de quienes buscan justicia por los feminicidios de sus hijas en Ciudad Juárez, quien visibilizó los impactos de la militarización; fue la voz extendida de las defensoras de derechos humanos en Chihuahua.

\section{Países y contexto social.}

Afganistán es considerado además uno de los países más peligrosos para los periodistas, y ocupa el puesto 122 de 180 en la clasificación mundial de la libertad de prensa 2020 de Reporteros Sin Fronteras (RSF).

Uno de los casos es el de la periodista Malalai Maiwand, quien fue asesinada el 10 de Diciembre de 2020, quien era una prominente defensora de los derechos de la mujer en un país como Afganistán. Hombres armados dispararon contra el coche de la periodista afgana, popular por su activismo social, particularmente en la defensa del derecho de las niñas a la educación.

Otro de los casos más extremos que nos ha tocado ser testigos, es el de las periodistas afganas Sadia Sadat, Shahnaz Roufi y Mursal Wahidi que perdieron su vida muy recientemente el 21 de Marzo de 2021. Las mismas, se encontraban en la lucha de ofrecer una voz discordante con el régimen del terrorismo.

Las amenazas tanto del grupo yihadista Estado Islámico (EI) como de los talibanes a los medios de comunicación se han repetido en las casi dos décadas de conflicto en Afganistán, perpetrando numerosos ataques contra la prensa, uno de los peores en enero de 2016 también contra Tolo, en el que murieron siete trabajadores.

Nouf Abdulaziz al-Jerawi es una periodista saudí que fue encarcelada por denunciar el sistema de tutela masculina en su país. En su prisión ha sido 
víctima de tortura y agresiones sexuales, sin que el Estado haya hecho nada para cuidarla.

Estas violencias a las mujeres, y al derecho a la vida y la integridad física se producen en el contexto de una cultura que tolera todas las formas de violencia contra las mujeres y que impide que las mujeres defensoras de los derechos humanos participen en espacios públicos tales como el periodismo y sean activistas. Las autoridades afganas deben garantizar su seguridad y poner fin a todas las formas de violencia contra las mujeres en el país.

\section{Ambiente de discriminación.}

Entonces, si ni las calles, ni las redes, son un espacio seguro para las mujeres periodistas, pero tampoco lo acaban de ser las oficinas internas de redacciones. En este caso, muchas periodistas fueron las primeras en denunciar casos de agresión o acoso sexual en sus lugares de trabajo con el movimiento \#Meetoo .

La periodista catalana Carlota Serra Llagostera, ha denunciado en su puesto de trabajo, acoso laboral e intentos de ejercicios de abuso de poder en su puesto de trabajo, por el hecho de ser mujer.

En Brasil, asimismo, la periodista Patricia Campos Mello ha pagado cara su investigación sobre el uso, por parte del presidente Bolsonaro, de fondos ilegales para financiar campañas de desinformación.

Así como también en Brasil, un grupo de cincuentas periodistas deportivas han puesto en marcha el movimiento \#DeixaElaTrabahlar (\#DejalaTrabajar) para denunciar los besos forzados a los que las someten los aficionados cuando cubren eventos deportivos en directo.

En Francia, cerca de 40 periodistas del diario deportivo L'Equipe se han unido para apoyar a sus colegas tras las denuncias de acoso en las "secciones de deportes “.

\section{Desigualdades y abusos.}

Así, podríamos continuar enumerando los abusos, desigualdades y penurias que las mujeres periodistas son sometidas todos los días dentro de esta estructura heteropatriarcal que nos encontramos viviendo.

La realidad es que la mujer es discriminada para ingresar o permanecer en su profesión de periodista, sea por su edad (muy joven/muy vieja). Cuando a los hombres se los elige por su contacto o por su inteligencia.

Por su tipo de cuerpo, muy gorda, de preferencias contratan a las mujeres por como lucen exteriormente. Por su tipo de cabello, de preferencia lacias. Cuando los hombres no tienen puesta su mirada en estas vanalidades.

Son examinadas y criticadas, por si repiten o no su atuendo, por poco o mucho maquillaje, son atacadas si se equivocan o cometen errores, por si llevan o no escote, si muestran sus curvas. Transformando a las periodistas en bustos parlantes.

Son atacadas por los temas en los que desean opinar, como por ejemplo el aborto, sexualidad, sexo, prostitución entre otras. Cuando los hombres siguen formando opinión y agenda sobre cómo tratar las temáticas.

También sesgadas por los temas que les asignan para opinar o presentar, bodas, modas, familias, cocina, entre otros. Cuando a los hombres se les asignan temas serios tales como la economía, la medicina, las informaciones importantes.

Otro punto es quienes abren el programa de noticias, siempre los hombres en Latinoamérica, en Europa, está ya normalizado.

Asimismo en las columnas siempre se publican las llevan las firmas de hombres. Hay mujeres columnistas, pero se invisibilizan. 


\section{Lenguaje y la mirada de las mujeres.}

El lenguaje crea mundo, crea realidades. Todas estas cuestiones previamente señaladas, son batallas y hay que darlas. Hay que deconstuir el modelo de que solo los hombres son la vanguardia de la intelectualidad, los formadores de opinión, y los que tienen más credibilidad en la información.

\section{Mujeres y su carrera profesional.}

Otra de las cuestiones que mas limita a las mujeres es la de tener hijos y su carrera de periodista.

Son las mujeres, casi exclusivamente, quienes, hasta la fecha, llevan a cuestas las tareas de cuidado que están repartidas así por cuestiones sexogenéricas, y de asignación de cuidado de la prole, o sea por el hecho de ser madres, se les asigno el lugar del hogar (privado), y doméstico.

FEDERICI (2013), la institución de la familia es esencialmente la institucionalización de nuestro trabajo no remunerado, de nuestra dependencia salarial de los hombres $\mathrm{y}$, consecuentemente, la institucionalización de la desigual división de poder que ha disciplinado tanto nuestras vidas como las de los hombres.

Para que se comprenda podemos decir que las multiplicidades de tareas que realiza y que debe realizar ya sea por mandato social, religioso, amoroso, patriarcal, o todo ello junto, son infinitas desde estar presente a toda hora, cuidar del hogar, cuidar de los niños, jugar con los niños, ahora con el Covid-19 hacer también de maestras y maestras de apoyo escolar, educar en valores y en ética a sus hijos, controlar o cuidar de los hijos para que nada les pase ( si algo sucede, "siempre" es culpa de la madre"), controlar horarios y hacer desayunos, almuerzos, meriendas, cenas, alimentar a toda la familia, dar de amamantar, bañar, lavar la ropa, coser, controlar el dinero de la familia, hablar amorosamente, generar amor, ser piadosas, hablar poco, hacer más, tener todo listo cuando el marido llega para que se sienta a gusto, atender a las visitas, y podría continuar hasta hacer un libro.

Las mujeres no conocemos ni siquiera nuestro propio valor, hacemos tanto, y ya lo tenemos tan arraigado en nuestra culturalidad, que la mujer media siquiera se cuestiona estos esquemas, sino es que se educa en ello. Con esto quiero decir, que la asignación de las tareas de cuidado a las mujeres, están en oposición con las del hombre, el cual no puede, no debe ni este obligado a hacerlas o colaborar en su concreción, debido a que simplemente no producen dinero en la economía, o al menos eso se pensaba.

AGUILAR (2019) nos indica, que estudiar el cuidado es problematizar la reproducción de la vida cotidiana y social. Participar del debate nos exige discutir cómo se resuelve la satisfacción de las necesidades y se sostiene la vida en el capitalismo $\mathrm{y}$ en sus potenciales alternativas. Instalar y discutir el cuidado en la agenda pública constituye un modo potente de politización de la domesticidad. Lo privado se vuelve público. Lo personal, político.

El Instituto de la Mujer en España editó un pequeño libro donde lista, y pone en valor todas estas tareas e intenta a su vez visibilizarlas manifestando que la perspectiva de género es esencial como respuesta al Covid-19.

En el Informe de la Comisión Interamericana de Derechos Humanos respecto a las mujeres y periodismo da cuenta de estos obstáculos, indicando que muchos de estos obstáculos y prácticas son manifestaciones de la discriminación basada en el género, además de otros factores, como la raza, el origen étnico, la religión o las creencias, la edad, la clase, la orientación sexual y la identidad de género, que sufren las mujeres en la región y que sigue generando disparidades en el ejercicio del derecho a la libertad de expresión en comparación con sus pares hombres. 
El impacto de estas prácticas discriminatorias es particularmente grave en el caso de mujeres que ejercen la libertad de expresión de manera activa y mantienen un alto perfil público, tales como las mujeres periodistas, las mujeres defensoras de derechos humanos y las mujeres políticas. Estos grupos de mujeres son doblemente atacados por ejercer la libertad de expresión y por su género. Además de los riesgos de amenazas y violencia que enfrentan todas las personas defensoras de derechos humanos y periodistas en la región, las mujeres que pertenecen a estos grupos están expuestas a riesgos adicionales o específicos. Al desafiar estereotipos machistas que reprueban su participación en la vida pública, se ven enfrentadas a la violencia y la discriminación contra las mujeres basada en su género, así como a formas diferenciadas de violencia por parte de actores estatales y no estatales.

A su vez, enfrentan desprotección y obstáculos en el acceso a la justicia, también diferenciados de sus compañeros varones.

\section{Los medios y los estereotipos.}

Los medios son grandes formadores de estereotipos. Los medios dominados por hombres crean esos estereotipos sobre las mujeres, cuerpos, vestimenta, opinión, entre otras.

Las mujeres reales tienen una mirada diferente. Le estamos faltando valor a la historia, cuando no hay igualdad y diversidad a la hora de invisibilizar las voces de las mujeres.

Hay que brindar referentes, para que la gente joven tenga claro que se puede ser mujer y decidir. Dar modelos reales. Buscando diversidad de fuentes como idóneas y profesionales. Para desnaturalizar esas desigualdades, brindando diversidad de géneros, cuerpos, religiones.

Para que las futuras generaciones puedan tener modelos de mujeres firmes realistas y empoderadas.

\section{Conclusiones}

En muchas o en casi todas las profesiones, por nombrar algunas, abogacía, periodismo, ingeniería, medicina, arquitectura, etc las mujeres que se dedican a las carreras serias, están invisibilizadas por el patriarcado.

Este patriarcado consta de los hombres, que generación tras generación elijen a sus discípulos, en muchos casos hijos, nietos, (hombres), para continuar no sólo en las titularidades de las redacciones, gerencias, etc., sino también para que sea y continúe el legado de su apellido y continúe siendo el más renombrado en determinada área.

El informe de la Comisión interamericana de Derechos Humanos, reafirma que las mujeres, en las todas las áreas, tienen que hacer opciones difíciles, postergar el matrimonio, maternidad. Para el caso de ser casada/en convivencia/ madre soltera y tener hijos, el patriarcado les he asignado el rol en base a la discriminación por el sexo, en donde debe ejercer el rol de cuidado para su propia familia y a veces la familia extendida. Entonces, hay que quedarse en el país y se quedan a estudiar o completar los estudios de posgrado, no están las condiciones, facilidades que permitan desarrollar los estudios sin una carga de trabajo que, a veces resulta, agobiante. La igualdad de oportunidades resulta ser una quimera cuando no se desmantela la discriminación estructural en contra de las mujeres. Tanto las mujeres periodistas como aquellas que trabajan en diferentes áreas de la comunicación deben lidiar con entornos amenazantes específicos que restringen su labor e impactan desproporcionadamente en el ejercicio de su derecho a la libertad de expresión.

Tanto en el sistema universal de los derechos humanos como en el sistema interamericano se han desarrollado normas y estándares que subrayan la obligación estatal de abordar los riesgos especiales $\mathrm{y}$ factores particulares que inhiben $\mathrm{u}$ obstaculizan el ejercicio del derecho a la libertad de expresión de las mujeres periodistas como parte de su obligación 
de respetar, proteger y garantizar el ejercicio de este derecho. Ello incluye la adopción de medidas positivas necesarias para la creación y mantenimiento de un entorno seguro y propicio para que las mujeres periodistas puedan ejercer su labor en condiciones de igualdad y sin discriminación y la necesidad de integrar una perspectiva de género en las políticas y medidas dirigidas a asegurar la seguridad de periodistas. Asimismo, los Estados tienen la obligación de proteger a las personas sometidas a su jurisdicción contra las injerencias indebidas de terceros en su derecho a la libertad de expresión, incluidas las empresas. Todas las empresas, incluidos los medios de comunicación y las plataformas en línea, tienen a su vez la responsabilidad de respetar los derechos humanos, a la luz de los estándares desarrollados en esta materia .

Desde la desigualdad en el ámbito laboral, los comentarios sexistas y misóginos, la violencia sexual o asesinatos de mujeres en razón de su género (o feminicidios), las amenazas o riesgos que enfrentan las mujeres periodistas tienden a ser invisibilizados y no son reconocidos como restricciones indebidas a la libertad de expresión por la mayoría de los colegas, medios de comunicación y autoridades estatales. A su vez, la desprotección de las mujeres periodistas frente a este tipo de violencia y las deficiencias en la investigación de estos crímenes impide que se atienda adecuadamente la particularidad de la situación de las mujeres periodistas y se garantice efectivamente el ejercicio de su derecho a la libertad de expresión.

La pandemia ha puesto por primera vez "en evidencia" el gran rol que ocupan las mujeres en la sociedad en cuanto a ejecutoras casi exclusivas de las tareas de cuidado. En esta crisis sanitaria, sea cuidado profesional o informal, se ha visibilizado como importante o al menos destacable. Se le ha agregado un pequeño valor moral o ético. Las tareas se han reasignado mínimamente, casi todo recae en la mujer, o en las hijas mujeres. El hombre solo se ocupaba de la compra o pasear al perro.
Soy de la idea, que la única manera de cambiar el mundo patriarcal en el que nuestras madres y abuelas han nacido es educarnos y educar a todas las mujeres que tenemos alrededor.

El cambio de paradigma, ya viene llegando, lento, pero a paso firme, hemos ganado conquistas, pero queda mucho aún por hacer en la mirada heteropatriarcal, sexista, que coloca a las mujeres en lugares físicos como una redacción o en lugares de internet, o en la calle en donde en todos ellos, son sometidas, silenciadas y hasta a veces violentadas psíquica y/o físicamente.

Asi como los enumera el informe de Periodismos y Sexismos manifiesta y solicita en cuatro guias de recomendaciones tanto a las redacciones solicitando mas igualdad y visibilidad tanto en los contenidos como en las condiciones de trabajo como asi también mas vigilancia y seguridad para las mujeres. A las mujeres periodistas recomendaciones respecto al ciberacoso, redacciones y viajes para cubrir hechos. A los Estados a que respeten la libertad de prensa, promover la igualdad, generar leyes y mecanismos para que las mujeres estén seguras en las redes, protegiendo y cuidando de la seguridad digital. Como así también, a las plataformas y a los anunciantes para que estén atentos a estas malas prácticas y no promuevan ni patrocinen campañas o eventos donde no exista paridad de géneros, igualdad de voces, entre otros.

Son los estados que deben conseguir lograr políticas públicas que den valor al trabajo silenciado e invisibilizado de las mujeres, para que tengan acceso a iguales garantías de acceso, permanencia y puedan competir a la par de los hombres.

Se deben generar conciencia, visibilizar las problemáticas y plantear mecanismos de asistencia, apoyo para destruir las barreras que impiden que las mujeres sean consideradas en igualdad de trato, valoración de sus ideas, flexibilización de normativas que coadyuven a su crecimiento en el rubro que han elegido perseverar, como así también mecanismos y 
protocolos para que las mismas se sientan seguras en sus casas, puestos de trabajo, en la red o en la calle al salir hacer su trabajo.

\section{Agradecimiento}

Artículo a ser incluido en el libro colectivo del I Congreso Internacional Libertad de Prensa en los Estados Democráticos, que se realizó los días 3 y 4 de junio de 2021. Línea temática: Eje 4. Prácticas periodísticas y Libertad de Prensa, de la cual he sido Ponente Internacional en fecha 4 de Junio 2021. Quiero agradecer al Dr. Dr. Eduardo Osorio (Delegado del Comité Científico) de referido Congreso, quien me ha honrado con su invitación a tan exitoso evento.

\section{Referencias}

Gabriela Nelba Guerrero Los derroteros del cuidado. Los derroteros del cuidado / ... [et al.]; compilado por Gabriela Nelba Guerrero ; Karina Inés Ramacciotti ; Marcela Zangaro. - 1a ed .Bernal: Universidad Nacional de Quilmes, 2019. Libro digital, PDF

Nicole Cox. Revolución en punto cero, Madrid, Traficantes de sueños, 2013. N. de. E.

Reporteros sin Fronteras. "El periodismo frente al sexismo", nuevo informe de RSF RFS-ES 8 de marzo de 2021. https://www.rsf-es.org/nuevoinforme-de-rsf-el-periodismo-frente-al-sexismo/

Unesco, Organización de las Naciones Unidas. Hechos y cifras Proyecto de supervisión de los medios de difusión a nivel mundial (GMMP) http://www.unesco.org/new/es/communicationand-information/crosscutting-priorities/genderand-media/women-make-the-news/facts-andfigures/

DW. El Mundo. La Unesco denuncia que el $73 \%$ de las periodistas sufre ciberacoso. América. JU (efe, en.unesco.org). 26.04.2021.https://www. dw.com/es/la-unesco-denuncia-que-el-73-de- las-periodistas-sufre-ciberacoso/a-57343245

Animal Político. Libertad de Expresión, mujeres y violencia. Por Blog Invitado 5 de junio, 2017 Atziri Ávila. Véase su nota en el siguiente link. https://www.animalpolitico.com/blog-invitado/ libertad-expresion-derecho-las-mujeres-vivirsin-violencia/

Federación de Sindicatos de Periodistas. Mursal Wahidi, Sadia Sadat y Shahnaz Roafi; asesinadas en Afganistán 3 marzo, 2021 https:// fesperiodistas.org/mursal-wahidi-sadia-sadat-yshahnaz-roafi-asesinadas-en-afganistan/

El País. Opinión. Tribuna El \#MeToo nació del mejor periodismo Los periodistas estadounidenses que han investigado minuciosamente los casos de abusos sexuales no perseguían el linchamiento público de los agresores sino una investigación factual EDUARDO SUÁREZ23 nov 2018-08:58 GMT https://elpais.com/elpais/2018/11/22/ opinion/1542908749_083853.html

CTXT. Contexto y Acción. Orgullosas de llegar tarde a las últimas noticias. Ser feminazi hoy. Hay mujeres columnistas No necesitamos que nos den voz, ya la tenemos, lo que tenemos que exigir son espacios para que se nos escuche. Es urgente. Anita Botwin 19 de Octubre de 2017 https:/ctxt.es/es/20171018/Firmas/15695/ mujeres-columnistas-medios-de-comunicacionmachismo-anita-botwin.htm

La perspectiva de género esencial en la respuesta a la COVID-19. Instituto de la Mujer, Ministerio de Igualdad. Secretaria de Estado de Igualdad y contra la violencia de género. Instituto de la Mujer para la Igualdad de Oportunidades. NIPO 049-20-024-8

Catálogo de publicaciones de la Administración General del Estado https://cpage.mpr.gob.es http://www.inmujer.gob.es/diseno/novedades/ IMPACTO_DE_GENERO_DEL_COVID_19_ 
(uv).pdf

RELE. Relatoría Especial para la Libertad de Expresión. Comisión Interamericana de Derechos Humanos. Mujeres Periodistas y Libertad de Expresión. OEA/SER.L/V/II CIDH/ RELE/INF.20/18 31 de octubre de 2018 Original: español. Mujeres Periodistas y Libertad de Expresión

Discriminación y violencia basada en el género contra las mujeres periodistas por el ejercicio de su profesión Relatoría Especial para la Libertad de Expresión de la Comisión Interamericana de Derechos Humanos.Edison Lanza.Relator Especial para la Libertad de Expresión. V. ; CM. (OAS. DOCUMENTOS OFICIALES ; OEA/ SER.L/V/II) ISBN 978-0-8270-6828-5 http:// www.oas.org/es/cidh/expresion/docs/informes/ MujeresPeriodistas.pdf

RELE. Relatoría Especial para la Libertad de Expresión. Comisión Interamericana de Derechos Humanos. Mujeres Periodistas y Libertad de Expresión. OEA/SER.L/V/II CIDH/ RELE/INF.20/18 31 de octubre de 2018 Original: español. Mujeres Periodistas y Libertad de Expresión

Discriminación y violencia basada en el género contra las mujeres periodistas por el ejercicio de su profesión Relatoría Especial para la Libertad de Expresión de la Comisión Interamericana de Derechos Humanos.Edison Lanza.Relator Especial para la Libertad de Expresión. V. ; CM. (OAS. DOCUMENTOS OFICIALES ; OEA/ SER.L/V/II) ISBN 978-0-8270-6828-5 https:// www.corteidh.or.cr/tablas/r39389.pdf

Reporteros sin Fronteras. 8 DE MARZO 2021- "El periodismo frente al sexismo", nuevo informe de RSF https://www.rsf-es.org/nuevo-informe-dersf-el-periodismo-frente-al-sexismo/ 